\title{
Dcx Re-expression Reduces Subcortical Band Heterotopia and Seizure Threshold in an Animal Model of Neuronal Migration Disorder
}

\author{
Jean-Bernard Manent ${ }^{1}$, Yu Wang ${ }^{1}$, YoonJeung Chang ${ }^{1}$, Murugan Paramasivam ${ }^{1}$, and \\ Joseph J LoTurco ${ }^{1}$ \\ ${ }^{1}$ Department of Physiology and Neurobiology, 75 North Eagleville Road U-3156, University of \\ Connecticut, Storrs, CT 06269, USA
}

\begin{abstract}
Disorders of neuronal migration can lead to malformations of the cerebral neocortex that greatly increase the risk of seizures. It remains untested whether malformations caused by disorders in neuronal migration can be reduced by reactivating cellular migration, and whether such repair can decrease seizure risk. Here we show, in a rat model of subcortical band heterotopia (SBH) generated by in utero RNAi of $D c x$, that aberrantly positioned neurons can be stimulated to migrate by re-expressing $D c x$ after birth. Re-starting migration in this way both reduces neocortical malformations and restores neuronal patterning. We find further that the capacity to reduce SBH has a critical period in early postnatal development. Moreover, intervention after birth reduces convulsant-induced seizure threshold to levels similar to that of malformation-free controls. These results suggest that disorders of neuronal migration may be eventually treatable by re-engaging developmental programs both to reduce the size of cortical malformations and to reduce seizure risk.
\end{abstract}

\section{Introduction}

A causal connection between disruptions in neuronal migration during fetal development and altered neocortical excitability is well established1,2,3,4,5. Foci of abnormally migrated neurons are particularly prevalent in patients with pharmacologically intractable epilepsies, and surgical resection of malformed cortex often can effectively treat such drug-resistant epilepsy6,7. Many cases, however, remain untreatable by surgery because of the location and/or widespread distribution of malformation(s). One such malformation occurs in double cortex syndrome in which a band of heterotopic grey matter composed of abnormally migrated neurons is located between the ventricular wall and the cortical mantle, and is separated from both by a band of white matter8,9. Focal resection of epileptogenic tissue in double cortex syndrome shows poor clinical outcome10. Double cortex syndrome or subcortical band heterotopia ( $\mathrm{SBH}$ ) is also associated with mild to moderate mental retardation 11 , intractable epilepsy in about $65 \%$ of patients 12 , and is most often caused in

Users may view, print, copy, and download text and data-mine the content in such documents, for the purposes of academic research, subject always to the full Conditions of use:http://www.nature.com/authors/editorial_policies/license.html\#terms

${ }^{1}$ Correspondence to: Joseph J Loturco e-mail: joseph.loturco@uconn.edu. 
female patients by mutation in the X-linked gene $D C X$, a microtubule binding protein essential to neuronal migration $13,14,15,16$. $D C X$ mutations in male patients usually cause predominantly anterior lissencephaly 15 but SBH associated with $D C X$ mutations have also been described in males 17.

Studies using animal models have revealed that several types of migration disruptions and malformations increase neuronal excitability and seizure risk. For example, spontaneous seizures are observed in the tish mutant rat18, and significantly reduced thresholds to convulsant agents are observed in rats with cortical migration anomalies caused by prenatal exposure to teratogens such as MAM19,20, cocaine21 or irradiation22. Similarly, in a freeze-lesion model of microgyria, epileptiform discharges are reliably evoked in brain slices containing malformations, and the threshold dose of convulsants to induce seizures is reduced23,24. A recent study also reported that spontaneous convulsive seizures can occasionally be observed in a subset of $D c x$ knockout mice showing discrete hippocampal malformations but no cortical abnormalities 25 . Together, results from animal models and studies on surgically removed human tissue indicate that malformed neocortex is associated with reorganized neuronal networks and altered cellular physiologies that create hyperexcitable tissue. It is currently unknown whether there is a time in development that interventions to reverse or reduce formed or forming malformations would also prevent neuronal hyperexcitability and seizure risk.

We previously developed a rat model of SBH by decreasing Dcx expression with in utero RNAi26. This model reproduces anatomical features of the malformations present in the human double cortex syndrome, and recently we have shown that the malformations are rescued or prevented by concurrent embryonic expression of Dcx27. Here we used a conditional variation of this rescue approach to determine whether delayed Dcx expression, after SBH have formed, can reduce heterotopia and restore neuronal patterning. We show that both laminar displacement of neurons and the size of SBH are reduced upon delayed expression of Dcx during early postnatal periods. We show further that animals with SBH are more susceptible to seizures induced by the convulsant PTZ, and that reduction of SBH restores seizure thresholds to levels similar to that of unaffected controls.

\section{Results}

\section{Conditional Re-expression of Dcx}

The purpose of the present study was to investigate whether neocortical lamination deficits and SBH malformations can be reduced by re-expression of Dcx after birth. Our approach was to initiate SBH formation and laminar displacement by in utero RNAi of $D c x$, and then re-express Dcx in mis-positioned neurons (Fig. 1a). In order to accomplish this we adapted the in vivo conditional transgene expression system developed by Matsuda and Cepko28 to a conditional RNAi rescue approach. Because endogenous Dcx expression decreases with neuronal development we could not perform conditional re-expression by gating "off" $D c x$ RNAi. Instead we created a system in which a version of $D c x$ that is insensitive to $D c x$ RNAi, was gated "on" in cells in which endogenous Dcx was knocked down by RNAi. To accomplish this we constructed a conditional DCX-eGFP expression vector (CALNL-DCXeGFP) which contains a stop codon flanked by two loxP sites downstream from the CAG 
promoter and upstream from DCX-EGFP sequence (Fig. 1b). The $D c x$ sequence in this plasmid vector is missing the 3'UTR of $D c x$, and the vector coding shRNA against $D c x$ (3UTRhp) that we developed previously26 targets the 3'UTR of $D c x$. Thus, unlike endogenous $D c x$, DCX-eGFP expressed from CALNL-DCX-eGFP is not targeted by $D c x$ RNAi. Another requirement of this strategy is that DCX-eGFP be expressed only after the addition of 4-OHT. To test for such controlled re-expression in vivo we transfected neocortical neuronal progenitors in utero at E14 with CALNL-DCX-eGFP, CAG$\mathrm{ER}^{\mathrm{T} 2} \mathrm{CreER}^{\mathrm{T} 2}$, CAG-mRFP, and 3UTRhp and injected pups with 4-OHT or vehicle control. In P15 neocortical sections from 4-OHT-injected rats, transfected cells were positive for eGFP signal and were immunopositive for Dcx (Fig. 1c). In addition, significant levels of DCX-eGFP expression were detected as early as 1 day after 4-OHT injection and peaked to over $80 \%$ of transfected cells after 2 days (Supplementary Fig. 1 online). In contrast, in litter-mate controls receiving the same in utero transfections, but injected with vehicle only, Dcx and eGFP expression were not detected. Thus, the system allows for temporally controlled re-expression of Dcx at times when expression of endogenous Dcx is decreased.

\section{Re-expression of Dcx Reduces SBH and Restores Laminar Position}

In order to determine when to first re-express Dcx in mis-positioned neurons we next established when SBH and lamination deficits were present after birth in the Dcx RNAi model26. Dcx knockdown caused laminar displacement and prominent SBH by the day of birth (P0) (Fig. 2a). Consequently, we conducted two types of experiments to induce reexpression of Dcx in mis-positioned neurons on P0 and analysed animals at P20. In the first, we controlled for the addition of 4-0HT and activity of Cre recombinase, and varied whether eGFP or DCX-eGFP was conditionally expressed respectively from CALNL-eGFP or CALNL-DCX-eGFP in animals electroporated at E14 with shRNA vectors targeting Dcx (Fig. 1d,e). This experiment controlled for possible effects of Cre recombinase as both groups received transfection of the plasmid CAG-ER ${ }^{\mathrm{T} 2} \mathrm{CreER}^{\mathrm{T} 2}$ and both received 4-OHT injections. We found that when DCX-eGFP was expressed at P0 SBH malformations were significantly reduced in size (Fig. 2b-e) relative to when eGFP was expressed. In addition, whereas transfected cells in the eGFP group accumulated in white matter and deep layers, transfected cells in the DCX-eGFP group were located predominantly in upper layers in a pattern similar to that found in animals that received a shRNA vector (3UTRm3hp) ineffective in decreasing Dcx expression (Fig. 2c). In a second version of the delayed rescue experiment we controlled for transfection of all plasmids, but varied whether animals in a litter received an injection of 4-OHT or vehicle solution (Fig. 3a). The results of this experiment were nearly identical to the first. SBH size assessed either by the density of neurons within the white-matter that were positive for the upper layer neuron marker CDP/ Cux1, or the cross sectional area of the malformation defined by accumulation of CDP/ $\mathrm{Cux} 1+$ neurons was significantly reduced in the group that received 4-OHT compared to the vehicle control group (Fig. 3b-d). In both types of re-expression experiments, DCX-eGFP expression caused a redistribution of transfected cells from aberrant positions to upper neocortical layers without a significant change in total number of cells. Together, these results indicate that cells normally fated for upper layers of neocortex but stalled at aberrant positions were induced by Dcx re-expression to migrate to their normally fated layer. 


\section{Rescued Neurons are Morphologically Similar to Normally Migrated Cells}

We next assessed whether the mis-positioned neurons induced to migrate to upper layers by Dcx re-expression were morphologically similar to neurons that migrated normally to their fated upper layer positions. We compared several morphological properties of apical dendrites in two conditions: a malformation free condition in which neurons were treated with an ineffective Dcx RNAi and were instead induced to express eGFP starting at P0, and a $\mathrm{P} 0$ rescue group in which neurons were initially arrested in SBH by Dcx RNAi and then stimulated to migrate by induced expression of DCX-eGFP (Fig. 4a-d). Labelled neurons in both groups were uniformly positive for the upper layer marker CDP/Cux1. In addition, there were no significant differences between the morphologies of labelled neurons in the two groups as assessed by number of dendritic processes (Fig. 4e), average length of processes (Fig. 4f) or total process length (Fig. 4g). In addition to normal dendritic development, rescued neurons extended axons across the corpus callosum and these axons projected to appropriate laminar territories in the contralateral hemisphere (Supplementary Fig. 2 online). We also found no significant difference in the number of interneruons in neocortex containing $\mathrm{SBH}$, in rescued neocortex, or in malformation-free contralateral hemispheres (Supplementary Fig. 3 and 4 online). These results indicate that rescued neurons stimulated to migrate away from aberrant positions settled in appropriate layers, continued to express laminar specific markers, and displayed appropriate dendritic and axonal differentiation patterns.

\section{Heterotopia Reduction Becomes Less Effective with Postnatal Development}

In order to identify the latest developmental time-point when induced Dcx re-expression could still rescue neocortical lamination and regress $\mathrm{SBH}$, we administered 4-OHT at P5 or $\mathrm{P} 10$ and compared the results to pups that received 4-OHT at P0. These three rescue treatments were also compared to animals that received only induction of eGFP at P0 (double cortex). The total number of cells, positions of transfected cells and size of SBH were analysed at P20 (Fig. 5). There was no significant difference in the total number of transfected cells/section between any condition indicating that there was no cell death induced by either eGFP or DCX-eGFP expression at P0, P5 or P10. In contrast, in both P5 and P10 rescue groups there was a significant shift of neurons towards upper layer positions relative to neurons in animals that received no rescue (Fig. 5f,g). Although neurons in the P5 and P10 rescue groups migrated further into upper layers than neurons in the un-rescued group, neurons rescued at P5 or P10 did not migrate as far into upper layers as did neurons that were rescued at P0 (Fig. 5f,g). The P5 rescue group, however, similar to the P0 rescue group had significantly smaller SBH than did the P10 rescue group or the group that received no rescue (Fig. 5h,i). These results indicate that re-expression of Dcx at P5 or P10 can induce partial recovery of neuronal position, that re-expression at $\mathrm{P} 5$ can regress $\mathrm{SBH}$, and that by P10 re-expression is no longer effective at reducing the size of SBH malformations.

\section{Reduction of SBH Reduces Seizure Susceptibility and Severity}

SBH in human patients are a well known risk factor for epilepsy11,12. We therefore, investigated whether the susceptibility to pentylenetetrazol (PTZ)-induced seizures was 
changed in animals with regressed SBH relative to unrescued animals. A series of subconvulsive doses of PTZ $(25 \mathrm{mg} / \mathrm{kg}$ ) were administrated intra-peritoneally every $10 \mathrm{~min}$ to animals in three different groups (described in Fig. 1d,e): one that had received 4-OHT to induce DCX-eGFP expression at P0 (P0 rescue), one that received 4-OHT at P0 to induce eGFP expression (double cortex, no rescue), and a third group that received ineffective shRNAs and so were free of malformations (malformation free). Injections of PTZ were continued until each animal displayed a generalized seizure. Seizure susceptibility and severity was quantified using the canonical Racine's scale of seizure severity including dose and latency to onset of the first minimal motor seizure and the first generalized tonic-clonic seizure (Fig. 6). Generalized seizures in the double cortex group occurred at significantly lower doses of PTZ (Fig. 6a) and with significantly shorter latencies (Fig. 6b) than either malformation free or P0 rescue animals. Importantly, malformation free and P0 rescue groups displayed similar seizure thresholds by all measures (Fig. $6 \mathrm{a}-\mathrm{c}$ ). These results suggest that Dcx re-expression at P0 restored levels of seizure risk back to levels of animals that had no neocortical malformation.

\section{Discussion}

This is the first study to our knowledge to demonstrate that a molecular intervention can reduce the size and functional effects of a pre-existing disruption in neuronal migration. In general, our study raises the possibility that in some contexts neuronal migration is a form of neuronal plasticity that may be engaged to induce neural repair. Previous examples of continued neuronal migration in the brain include neuronal migration of endogenous neurons to the olfactory bulb and dentate gyrus throughout life29, as well as migration of transplanted neuronal progenitors into neocortex and other regions of CNS30. Our study extends this phenomenon of continued migration to include induced migration of mispositioned neurons in neocortex. Our study also suggests that reversing a migration disruption in development can reduce aberrant excitability levels in more mature brain.

In this study we demonstrate that there are significant developmental constraints on stimulating migration in the postnatal neocortex by re-expression of Dcx. Whereas Dcx reexpression at $\mathrm{P} 0$ induced a migration that significantly regressed heterotopia and restored neocortical lamination, re-expression at P5 led to partial restoration of neuronal position with heterotopia regression, and re-expression at P10 led to partial recovery of position without significant heterotopia reduction. These ages in rats correspond roughly in humans to preterm gestational weeks, 25-35 for P0 to several months after birth for P10. We do not currently know the reason for absence of regression at P10 or the reduced migration following P5 intervention. It is likely, however, that developmental changes in neocortical tissue and within differentiating neurons limit continued neuronal migration into the postnatal period. Migration of pyramidal neurons during normal neocortical development is guided by radial glial processes 31 , and it is likely that developmental loss of radial glia, which normally occurs by the middle of the second postnatal week in the rat, reduces the potential for continued migration32,33. In addition, the developmental window for migration out of SBH may close because of neuronal maturation. Neurons within SBH form synapses and send axonal projections to subcortical targets 26,27 , and such neuronal differentiation may preclude induced migration by re-expression of Dcx. It is important to 
note, however, that even as late as P10 our results show that there is still a capacity for plasticity, and that mis-positioned neurons can be shifted from deeper to more superficial positions by expression of Dcx. In the future it will be important to determine which specific cellular changes are responsible for developmental restriction of induced re-migration, and whether these can be manipulated to allow for heterotopia regression in even more mature animals.

Virtually all neuronal migration disorders including lissencephaly, periventricular nodular heterotopias, and subcortical band heterotopia are risk factors for seizures34,35,36. In addition, focal cortical dysplasias (FCDs) are estimated to be associated with $30 \%$ of intractable temporal lobe epilepsies 37,38 . The specific mechanisms that cause dysplastic cortices to cause epileptiform activity remain unclear. It has remained unknown for example when in development the presence of a neocortical malformation first disposes cortical tissue to overexcitability $39,40,1,35,41$. Our results suggest that an underlying cause of seizure susceptibility to reduced PTZ dose is directly related to aberrant neuronal positioning and to the malformation. We can not yet distinguish from our results at what point in development migration impairment causes increased excitability, but regression initiated following birth is sufficient to restore PTZ thresholds to control levels. Future experiments can now be directed at determining the time course of changes in synaptic and cellular physiology that correlate with reduced seizure susceptibility.

The specific experimental intervention we used to restore migration patterns, electroporation of an inducible construct, is clearly not applicable to human patients. There are at least two possible directions indicated by our findings for interventions that may one day be therapeutically viable. First, Dcx expression may be induced by viral transduction of cells within SBH malformations. Our results currently suggest that such gene therapy would probably need to be applied perinatally, but could be restricted to mis-positioned neurons within SBH. An alternative to gene therapy could be to enhance the function of other members of the $D C X$ superfamily that may have redundant function with DCX. For example, in mouse knockouts of $D c x$ there are no cortical dysplasias42, and only when Dcx and Dclk are both disrupted do migration disorders similar to human lissencephaly appear43. Thus Dclk appears in the mouse to be capable of compensating for Dcx function. There are now multiple defined members of the $D C X$ super-family with similar cellular and biochemical functions44, and perhaps pharmacological enhancement of the function of one or several of these may facilitate regression of SBH in humans. Finally, the present study serves as a proof of concept that mis-migrated cells can be manipulated to restore a normal morphological pattern and level of neuronal excitability in the cerebral cortex.

\section{Methods}

\section{In utero electroporation and Dcx RNAi}

In utero electroporation was performed as described before 26 . We used shRNAs targeting the 3' UTR of Dcx (3UTRhp) or an ineffective shRNA with 3 point mutations creating mismatches (3UTRm3hp). We previously showed26 that 3UTRhp construct efficiently knocks-down Dcx expression in vivo whereas 3UTRm3hp construct is ineffective to cause $D c x$ knock-down. In addition, Dcx RNAi-induced phenotype is rescued by Dcx expression, 
confirming the specificity of shRNAs27. Plasmids coding mRFP (CAG-mRFP, $0.5 \mu \mathrm{g} \mathrm{ll}^{-1}$ ) to fluorescently label transfected cells were co-electroporated with plasmids expressing shRNAs against $D c x$ (3UTRhp or 3UTRm3hp, $0.5 \mu \mathrm{g} \mu \mathrm{l}^{-1}$ ), a 4-OHT-activatable form of Cre Recombinase (CAG-ER ${ }^{\mathrm{T} 2} \mathrm{CreER}^{\mathrm{T} 2}, 1.0 \mu \mathrm{g} \mu \mathrm{l}^{-1}$ ) and Cre-dependent inducible expression vectors (CALNL-eGFP, $1.5 \mu \mathrm{g}^{-1}$ ) (a gift from T. Matsuda and C. Cepko, Harvard Medical School, Boston) and the plasmid CALNL-DCX-eGFP $\left(1.5 \mu \mathrm{g} \mu \mathrm{l}^{-1}\right)$ which was made for this study. A pCAGGS-DCX-eGFP plasmid was constructed based on previously described pCAGGS-DCX plasmid 27 by fusing eGFP at the C-terminus. To generate CALNL-DCX-eGFP, the DCX-eGFP fusion fragment from pCAGGS-DCX-eGFP was subcloned into EcoRI and NotI sites of CALNL-eGFP plasmid. All animal protocols were approved by the University of Connecticut Institutional Animal Care and Use Committee.

\section{4-OHT administration}

4-hydroxytamoxifen (4-OHT; Sigma) was dissolved in 95\% ethanol at a concentration of 20 $\mathrm{mg} \mathrm{ml}^{-1}$ and diluted with 9 volumes of corn oil. Diluted 4-OHT ( $2 \mathrm{mg} / 100 \mathrm{~g}$ body weight) was administered to the animals via i.p. injections. Vehicle-treated animals were injected with the same solution without 4-OHT.

\section{Histological procedures and microscopy}

Animals were transcardially perfused under deep anaesthesia with $4 \%$ paraformaldehyde in PBS. Brains were removed and post-fixed $48 \mathrm{~h}$ in the same fixative solution, prior to frontal sectioning with a vibratome (Leica, Nussloch, Germany). Brain sections were processed for immunohistochemistry as floating sections. Primary antibodies were: goat anti-doublecortin (1:100, Santa Cruz Biotechnology, inc.), rabbit anti-CDP/Cux1 (1:200, Santa Cruz Biotechnology, inc.), mouse and rabbit anti-GFP (1:2000 and 1:3000, Molecular Probes), rabbit anti-RFP (1:3000, Chemicon). Appropriate secondary antibodies (1:200) conjugated with Alexa 488, 568, 633 (Chemicon) or Cy5 (Jackson ImmunoResearch, inc.) were used and sections were counterstained with the fluorescent Nissl staining NeuroTrace 435/455 (1:200, Molecular Probes).

Photomicrographs were taken using a Nikon Eclipse E400 microscope (Tokyo, Japan) equipped with a digital Spot Camera (Diagnostic Instruments, USA) or with a Leica TCS SP2 confocal microscope (Nussloch, Germany).

\section{Seizure induction with PTZ}

Pentylenetetrazole (PTZ; Sigma) was dissolved in $0.9 \%$ saline and administered to P30 animals via i.p. injections at a concentration of $25 \mathrm{mg}$ per $\mathrm{kg}$ of body weight. There was no statistically significant difference in weight or sex-ratio between groups of animals. Rats were placed in Plexiglass cages, observed by an experimenter naïve to the status of the animal and their behaviour was scored using the Racine's scale45. PTZ was administered every 10 minutes until generalized seizures occurred. 


\section{Quantifications and statistical analyses}

Quantifications were performed with the image analysis software ImageJ 1.39e (Wayne Rasband, NIH, USA) on coronal sections $(60 \mu \mathrm{m})$ located from Bregma -3.00 to Bregma -5.00 according to the atlas of Paxinos and Watson46. Relative positions of transfected cells in the cortex were quantified by counting mRFP+ cells in 12 areas of interests normalized in individual sections to fit within the whole thickness of the cortical wall, from deep to superficial layers excluding the white matter. Size of SBH was quantified in square pixels using ImageJ measuring tools. Morphological analyses of dendritic arborization were performed after neuronal reconstructions with the ImageJ plug-in NeuronJ 1.2 (Erik Meijering).

Statistical tests were performed using InStat 3.0 (GraphPad). Normality of the data distribution was checked using Kolmogorov and Smirnov method. We used two-sample Student's t-test to compare means of 2 independent groups. When 3 groups of data were compared, we used one-way ANOVA with Tukey-Kramer post-test for multiple comparisons. We considered values as significant when $P<0.05$. All data are presented \pm s.e.m.

\section{Supplementary Material}

Refer to Web version on PubMed Central for supplementary material.

\section{Acknowledgments}

This work was supported by the National Institute of Health (MH056524 and NS062416 to JJL) and the Jerome Lejeune foundation (research fellowship to JBM).

\section{References}

1. Jacobs KM, Kharazia VN, Prince DA. Mechanisms underlying epileptogenesis in cortical malformations. Epilepsy Res. 1999; 36:165-188. [PubMed: 10515164]

2. Chevassus-au-Louis N, Baraban SC, Gaïarsa JL, Ben-Ari Y. Cortical malformations and epilepsy: new insights from animal models. Epilepsia. 1999; 40:811-821. [PubMed: 10403203]

3. Schwartzkroin PA, Walsh CA. Cortical malformations and epilepsy. Ment Retard Dev Disabil Res Rev. 2000; 6:268-280. [PubMed: 11107192]

4. Kuzniecky RI, Barkovich AJ. Malformations of cortical development and epilepsy. Brain Dev. 2001; 23:2-11. [PubMed: 11226722]

5. Sisodiya SM. Malformations of cortical development: burdens and insights from important causes of human epilepsy. Lancet Neurol. 2004; 3:29-38. [PubMed: 14693109]

6. Sisodiya SM. Surgery for malformations of cortical development causing epilepsy. Brain. 2000; 123(Pt 6):1075-1091. [PubMed: 10825348]

7. Guerrini R. Genetic malformations of the cerebral cortex and epilepsy. Epilepsia. 2005; 46(Suppl 1): 32-37. [PubMed: 15816977]

8. Barkovich AJ, Jackson DEJ, Boyer RS. Band heterotopias: a newly recognized neuronal migration anomaly. Radiology. 1989; 171:455-458. [PubMed: 2468173]

9. Dobyns WB, Andermann E, Andermann F, Czapansky-Beilman D, Dubeau F, Dulac O, et al. Xlinked malformations of neuronal migration. Neurology. 1996; 47:331-339. [PubMed: 8757001]

10. Bernasconi A, Martinez V, Rosa-Neto P, D'Agostino D, Bernasconi N, Berkovic S, et al. Surgical resection for intractable epilepsy in double cortex syndrome yields inadequate results. Epilepsia. 2001; 42:1124-1129. [PubMed: 11580758] 
11. Barkovich AJ, Guerrini R, Battaglia G, Kalifa G, N'Guyen T, Parmeggiani A, et al. Band heterotopia: correlation of outcome with magnetic resonance imaging parameters. Ann. Neurol. 1994; 36:609-617. [PubMed: 7524438]

12. Guerrini R, Carrozzo R. Epilepsy and genetic malformations of the cerebral cortex. Am. J. Med. Genet. 2001; 106:160-173. [PubMed: 11579436]

13. Pinard JM, Motte J, Chiron C, Brian R, Andermann E, Dulac O. Subcortical laminar heterotopia and lissencephaly in two families: a single X linked dominant gene. J. Neurol. Neurosurg. Psychiatr. 1994; 57:914-920. [PubMed: 8057113]

14. des Portes V, Francis F, Pinard JM, Desguerre I, Moutard ML, Snoeck I, et al. doublecortin is the major gene causing X-linked subcortical laminar heterotopia (SCLH). Hum. Mol. Genet. 1998; 7:1063-1070. [PubMed: 9618162]

15. Gleeson JG, Allen KM, Fox JW, Lamperti ED, Berkovic S, Scheffer I, et al. Doublecortin, a brainspecific gene mutated in human X-linked lissencephaly and double cortex syndrome, encodes a putative signaling protein. Cell. 1998; 92:63-72. [PubMed: 9489700]

16. Matsumoto N, Leventer RJ, Kuc JA, Mewborn SK, Dudlicek LL, Ramocki MB, et al. Mutation analysis of the DCX gene and genotype/phenotype correlation in subcortical band heterotopia. Eur. J. Hum. Genet. 2001; 9:5-12. [PubMed: 11175293]

17. D'Agostino MD, Bernasconi A, Das S, Bastos A, Valerio RM, Palmini A, et al. Subcortical band heterotopia (SBH) in males: clinical, imaging and genetic findings in comparison with females. Brain. 2002; 125:2507-2522. [PubMed: 12390976]

18. Lee KS, Schottler F, Collins JL, Lanzino G, Couture D, Rao A, et al. A genetic animal model of human neocortical heterotopia associated with seizures. J. Neurosci. 1997; 17:6236-6242. [PubMed: 9236234]

19. Chevassus-Au-Louis N, Rafiki A, Jorquera I, Ben-Ari Y, Represa A. Neocortex in the hippocampus: an anatomical and functional study of CA1 heterotopias after prenatal treatment with methylazoxymethanol in rats. J. Comp. Neurol. 1998; 394:520-536. [PubMed: 9590559]

20. Baraban SC, Schwartzkroin PA. Flurothyl seizure susceptibility in rats following prenatal methylazoxymethanol treatment. Epilepsy Res. 1996; 23:189-194. [PubMed: 8739122]

21. Baraban SC, McCarthy EB, Schwartzkroin PA. Evidence for increased seizure susceptibility in rats exposed to cocaine in utero. Brain Res. Dev. Brain Res. 1997; 102:189-196. [PubMed: 9352101]

22. Roper SN, Gilmore RL, Houser CR. Experimentally induced disorders of neuronal migration produce an increased propensity for electrographic seizures in rats. Epilepsy Res. 1995; 21:205219. [PubMed: 8536674]

23. Jacobs KM, Gutnick MJ, Prince DA. Hyperexcitability in a model of cortical maldevelopment. Cereb. Cortex. 1996; 6:514-523. [PubMed: 8670677]

24. Luhmann HJ, Raabe K. Characterization of neuronal migration disorders in neocortical structures: I. Expression of epileptiform activity in an animal model. Epilepsy Res. 1996; 26:67-74. [PubMed: 8985688]

25. Nosten-Bertrand M, Kappeler C, Dinocourt C, Denis C, Germain J, Phan Dinh Tuy F, et al. Epilepsy in Dcx knockout mice associated with discrete lamination defects and enhanced excitability in the hippocampus. PLoS ONE. 2008; 3:e2473. [PubMed: 18575605]

26. Bai J, Ramos RL, Ackman JB, Thomas AM, Lee RV, LoTurco JJ. RNAi reveals doublecortin is required for radial migration in rat neocortex. Nat. Neurosci. 2003; 6:1277-1283. [PubMed: 14625554]

27. Ramos RL, Bai J, LoTurco JJ. Heterotopia formation in rat but not mouse neocortex after RNA interference knockdown of DCX. Cereb. Cortex. 2006; 16:1323-1331. [PubMed: 16292002]

28. Matsuda T, Cepko CL. Controlled expression of transgenes introduced by in vivo electroporation. Proc. Natl. Acad. Sci. U.S.A. 2007; 104:1027-1032. [PubMed: 17209010]

29. Lim DA, Huang Y, Alvarez-Buylla A. The adult neural stem cell niche: lessons for future neural cell replacement strategies. Neurosurg. Clin. N. Am. 2007; 18:81-92. ix. [PubMed: 17244556]

30. Imitola J, Raddassi K, Park KI, Mueller F, Nieto M, Teng YD, et al. Directed migration of neural stem cells to sites of CNS injury by the stromal cell-derived factor 1alpha/CXC chemokine receptor 4 pathway. Proc. Natl. Acad. Sci. U.S.A. 2004; 101:18117-18122. [PubMed: 15608062] 
31. Rakic P. Mode of cell migration to the superficial layers of fetal monkey neocortex. J. Comp. Neurol. 1972; 145:61-83. [PubMed: 4624784]

32. Stichel CC, Muller CM, Zilles K. Distribution of glial fibrillary acidic protein and vimentin immunoreactivity during rat visual cortex development. J. Neurocytol. 1991; 20:97-108. [PubMed: 2027041]

33. Kalman M, Ajtai BM. A comparison of intermediate filament markers for presumptive astroglia in the developing rat neocortex: immunostaining against nestin reveals more detail, than GFAP or vimentin. Int. J. Dev. Neurosci. 2001; 19:101-108. [PubMed: 11226759]

34. Guerrini R, Canapicchi R, Dobyns WB. Epilepsy and malformations of the cerebral cortex. Neurologia. 1999; 14(Suppl 3):32-47. [PubMed: 10379165]

35. Baraban S. Epileptogenesis in the Dysplastic Brain: A Revival of Familiar Themes. Epilepsy Curr. 2001; 1:6-11. [PubMed: 15309030]

36. Sheen VL, Walsh CA. Developmental genetic malformations of the cerebral cortex. Curr Neurol Neurosci Rep. 2003; 3:433-441. [PubMed: 12914687]

37. Crino PB. Malformations of cortical development: molecular pathogenesis and experimental strategies. Adv. Exp. Med. Biol. 2004; 548:175-191. [PubMed: 15250594]

38. Cepeda C, Andre VM, Flores-Hernandez J, Nguyen OK, Wu N, Klapstein GJ, et al. Pediatric cortical dysplasia: correlations between neuroimaging, electrophysiology and location of cytomegalic neurons and balloon cells and glutamate/GABA synaptic circuits. Dev. Neurosci. 2005; 27:59-76. [PubMed: 15886485]

39. Chae T, Kwon YT, Bronson R, Dikkes P, Li E, Tsai LH. Mice lacking p35, a neuronal specific activator of Cdk5, display cortical lamination defects, seizures, and adult lethality. Neuron. 1997; 18:29-42. [PubMed: 9010203]

40. Hablitz JJ, DeFazio T. Excitability changes in freeze-induced neocortical microgyria. Epilepsy Res. 1998; 32:75-82. [PubMed: 9761310]

41. Andres M, Andre VM, Nguyen S, Salamon N, Cepeda C, Levine MS, et al. Human cortical dysplasia and epilepsy: an ontogenetic hypothesis based on volumetric MRI and NeuN neuronal density and size measurements. Cereb. Cortex. 2005; 15:194-210. [PubMed: 15297365]

42. Corbo JC, Deuel TA, Long JM, LaPorte P, Tsai E, Wynshaw-Boris A, et al. Doublecortin is required in mice for lamination of the hippocampus but not the neocortex. J. Neurosci. 2002; 22:7548-7557. [PubMed: 12196578]

43. Koizumi H, Tanaka T, Gleeson JG. Doublecortin-like kinase functions with doublecortin to mediate fiber tract decussation and neuronal migration. Neuron. 2006; 49:55-66. [PubMed: 16387639]

44. Coquelle FM, Levy T, Bergmann S, Wolf SG, Bar-El D, Sapir T, et al. Common and divergent roles for members of the mouse DCX superfamily. Cell Cycle. 2006; 5:976-983. [PubMed: 16628014]

45. Racine RJ. Modification of seizure activity by electrical stimulation. I. After-discharge threshold. Electroencephalogr Clin Neurophysiol. 1972; 32:269-279. [PubMed: 4110396]

46. Paxinos, G.; Watson, C. The rat brain in stereotaxic coordinates. In: Elsevier, editor. Elsevier Academic Press; 2005. 
a

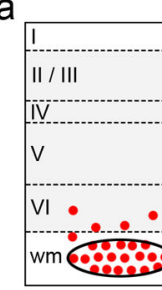

Dcx off

d
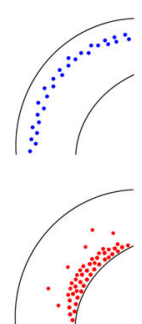

b

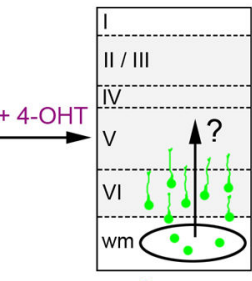

Dcx on

Malformation free 3UTRm3hp CALNL-eGFP CAG-ER ${ }^{T 2} C r e E R^{T 2}$ CAG-mRFP

Double cortex 3UTRhp CALNL-eGFP CAG-ER ${ }^{\top 2} C r e E R^{\top 2}$ CAG-mRFP

P0 / P5 / P10 rescue 3UTRhp

CALNL-DCX-eGFP

CAG-ER ${ }^{\text {T2 }} \mathrm{CreER}^{\text {T2 }}$

CAG-mRFP

e
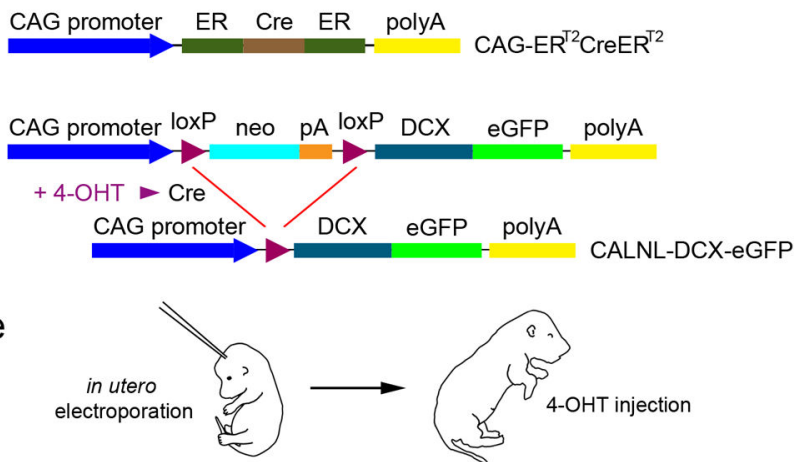

Dcx knockdown

\begin{tabular}{ccccc} 
& \multicolumn{1}{c}{ Dcx knockdown } & 4-OHT-induced expression \\
& E14 & P0 & P5 & P10 \\
Malformation free & $\times$ & eGFP & \\
Double cortex & $\checkmark$ & eGFP & \\
P0 rescue & $\checkmark$ & DCX-eGFP & \\
P5 rescue & $\checkmark$ & \multicolumn{3}{c}{ DCX-eGFP } \\
P10 rescue & $\checkmark$ & & & DCX-eGFP
\end{tabular}

C

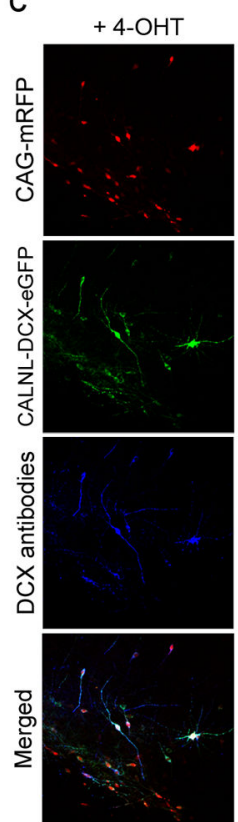

Vehicle

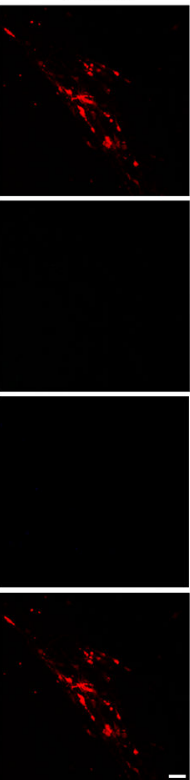

Figure 1. Temporal control of Dcx expression in mis-positioned neurons by using Cre-dependent expression vectors and 4-OHT-activatable Cre recombinase

(a) A schematic of the experimental approach used to test the hypothesis that re-expression of Dcx can reactivate migration and regress malformation. (b) A 4-OHT-activatable Cre recombinase composed of two ER binding domains is expressed under the control of the CAG promoter (CAG-ER ${ }^{\mathrm{T} 2} \mathrm{CreER}^{\mathrm{T} 2}$ ). Cre-dependent Dcx expression vector contains neo with a stop codon flanked by loxP sites (CALNL-DCX-eGFP). In the presence of 4-OHT, recombination occurs and DCX-eGFP is expressed. (c) Animals were electroporated at E14 with 4 plasmids: CAG-mRFP, CAG-ER ${ }^{\mathrm{T} 2} \mathrm{CreER}^{\mathrm{T} 2}$, CALNL-DCX-eGFP and 3UTRhp. In 4-OHT-treated animals (left panels), DCX-GFP is expressed and Dcx is detected with antibodies in transfected mis-positioned neurons. In vehicle-treated animals (right panels), no signal is detected in the green channel or with Dcx antibodies. (d) Transfection conditions. (e) Scheme of experiments. Scale bars, 50 $\mathrm{mm}$. 
a E14 : P0

E14 : P5

E14 : P10

E14 : P15

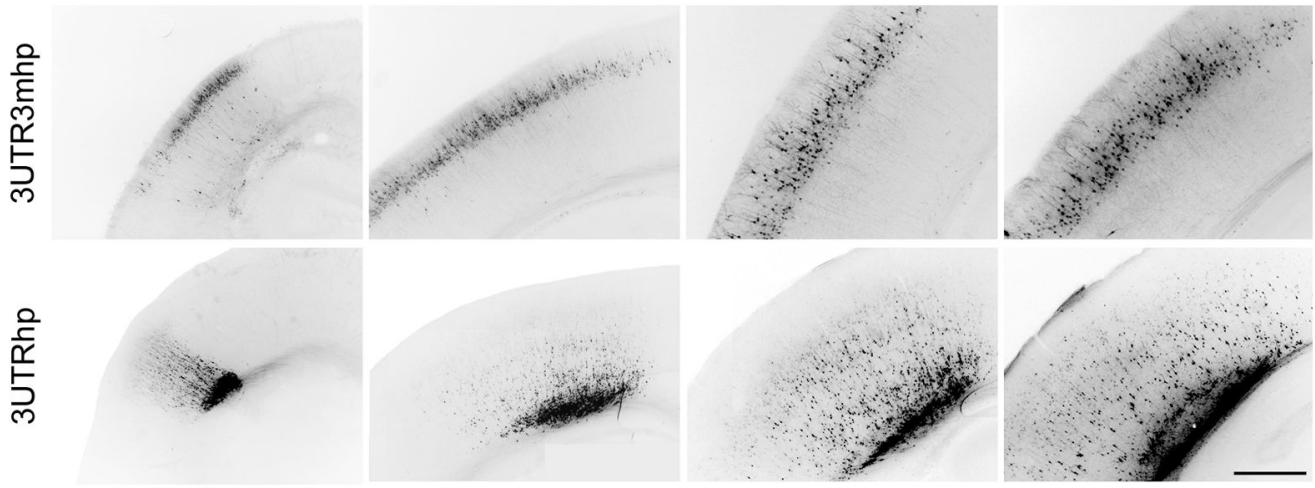

b

CAG-mRFP / 3UTRhp

CALNL-DCX-eGFP

Merged (+ Blue Nissl)
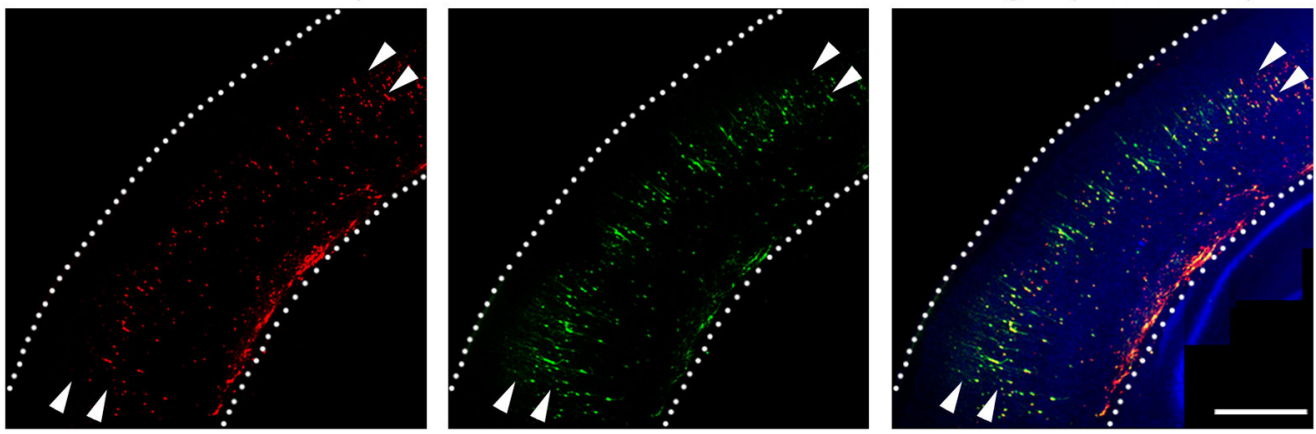

C

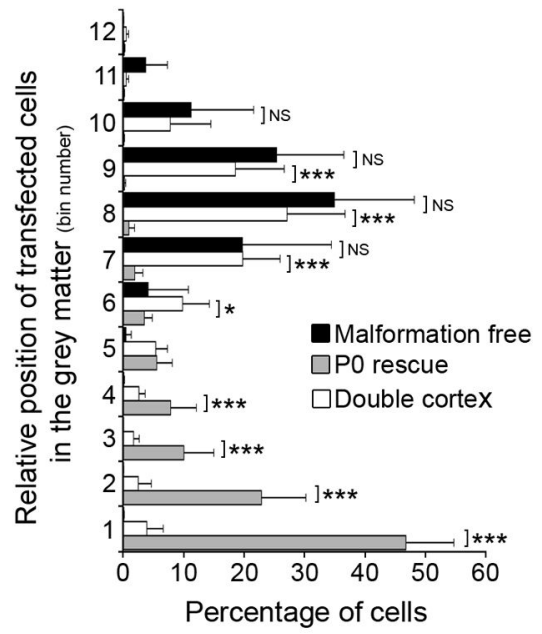

d

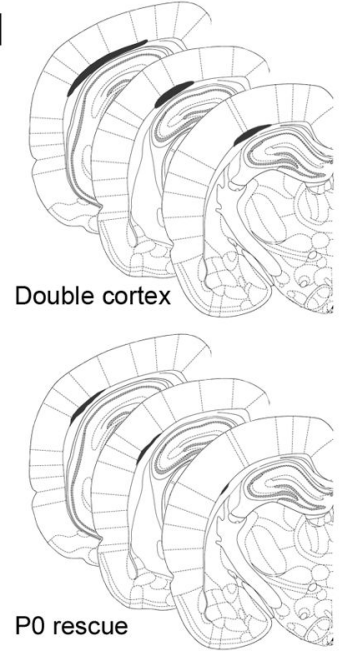

e

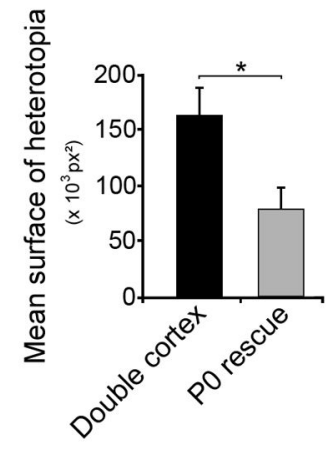

Figure 2. Restoration of neocortical lamination and regression of SBH after re-expression of Dcx at P0

(a) Representative frontal neocortical sections showing laminar position of transfected cells in animals electroporated at E14 with either effective (3UTRhp) (bottom panels) or noneffective (3UTRm3hp) (top panels) DCX-targeting shRNA vectors together with CAGmRFP, and sacrificed postnatally from P0 to P15. (b) Representative frontal neocortical section showing restoration of neocortical lamination at P20 after re-expression of Dcx in mis-positioned neurons at P0. 4 plasmids were electroporated at E14: CAG-mRFP, CAG- 
ER $^{\mathrm{T} 2} \mathrm{CreER}^{\mathrm{T} 2}$, CALNL-DCX-eGFP and 3UTRhp, and 4-OHT was administered at birth. (c) Quantification of transfected cells distribution within the neocortical grey matter after induction of eGFP or DCX-eGFP expression in animals electroporated at E14 with either effective (3UTRhp) or non-effective (3UTRm3hp) $D c x$-targeting shRNA vectors, together with CA-mRFP, CAG-ER ${ }^{\mathrm{T} 2} \mathrm{CreER}^{\mathrm{T} 2}$, and CALNL-DCX-eGFP or CALNL-eGFP (10-12 sections from 2-3 animals per condition). (d) Size and position of SBH at 3 rostro-caudal levels after induction of eGFP (top) or DCX-eGFP (bottom) expression in animals electroporated at E14 with CAG-mRFP, CAG-ER ${ }^{\mathrm{T} 2} \mathrm{CreER}^{\mathrm{T} 2}$, 3UTRhp, and CALNL-eGFP (top) or CALNL-DCX-eGFP (bottom).

(e) Quantification of SBH surface after induction of eGFP or DCX-eGFP in the same experimental conditions (8-9 sections from $2-3$ animals per condition). $* * * P<0.001$, $* P$ $<0.05$. Scale bars, $500 \mu \mathrm{m}(\mathbf{a}, \mathbf{b})$ 
a

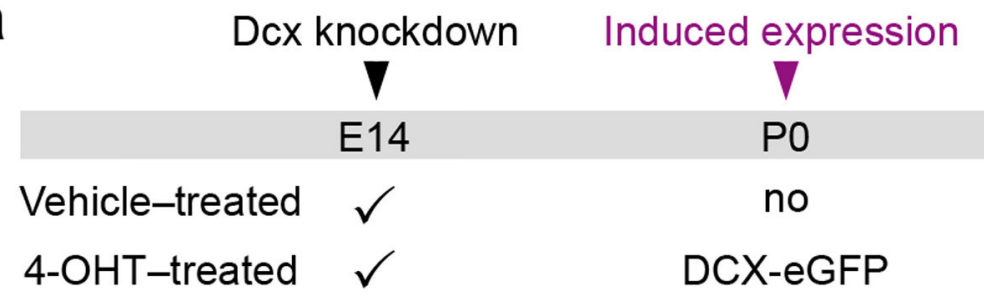

b

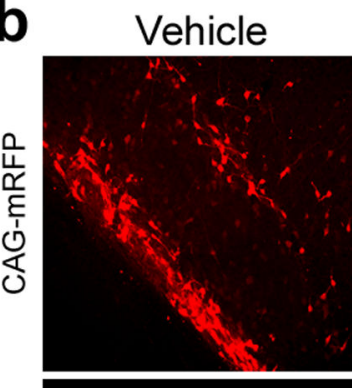

虽
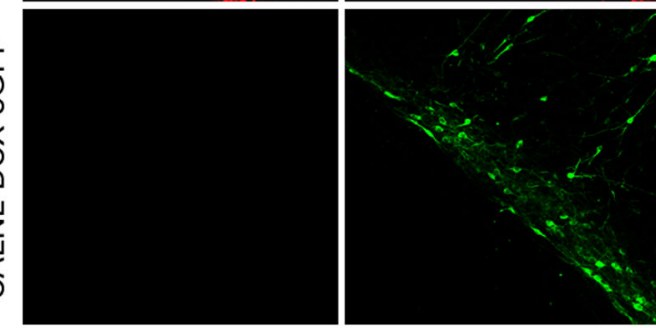

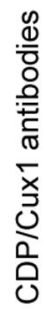
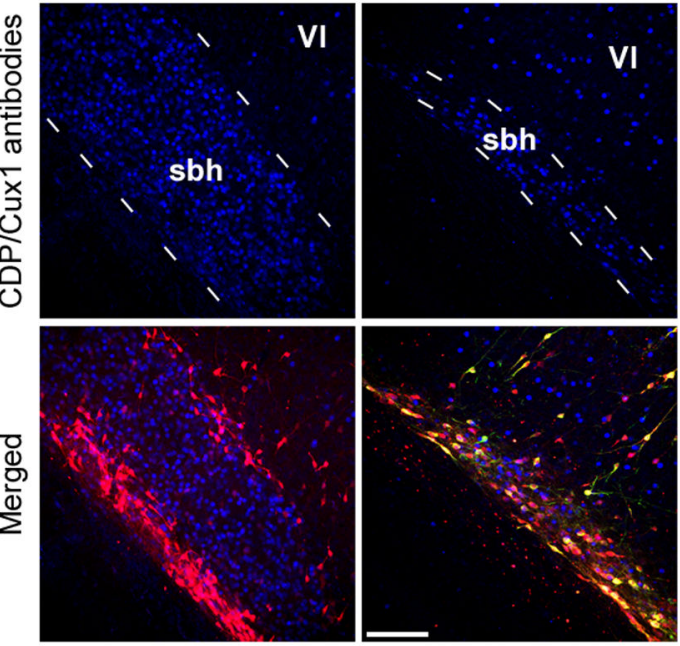
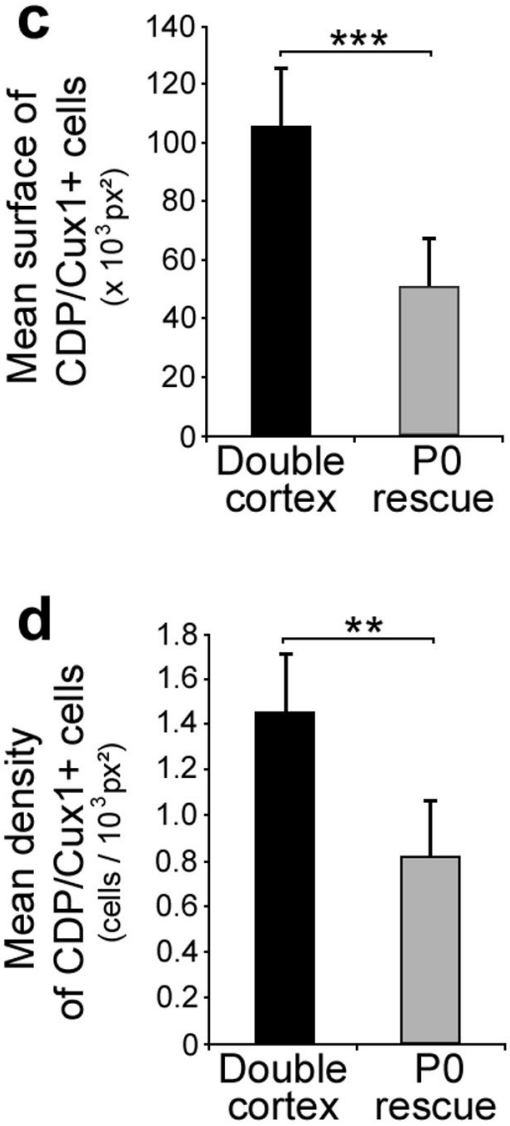

Figure 3. Migration of Fated Upper Layer neurons from SBH

(a) A scheme of experiment. Four plasmids were electroporated at E14: CAG-mRFP, CAG$\mathrm{ER}^{\mathrm{T} 2} \mathrm{CreER}^{\mathrm{T} 2}$, CALNL-DCX-eGFP and 3UTRhp, and 4-OHT or its vehicle solution was administered at birth. DCX-eGFP expression is induced in the 4-OHT-treated group only. (b) Immunohistochemistry of P20 neocortical sections showing E14 transfected and nontransfected cells immunopositive for the upper layers neurons marker CDP/Cux1 (d, h) within SBH at P20, in the absence of Dcx re-expression (left panels), or after Dcx reexpression (right panels). 
(c-d) Quantifications of surface (c) and density (d) of CDP/Cux 1+ cells within SBH in the same experimental conditions (6 sections from 2 animals per condition). *** $P<0.001$, ** $P<0.01$. Scale bar, $150 \mu \mathrm{m}$. 

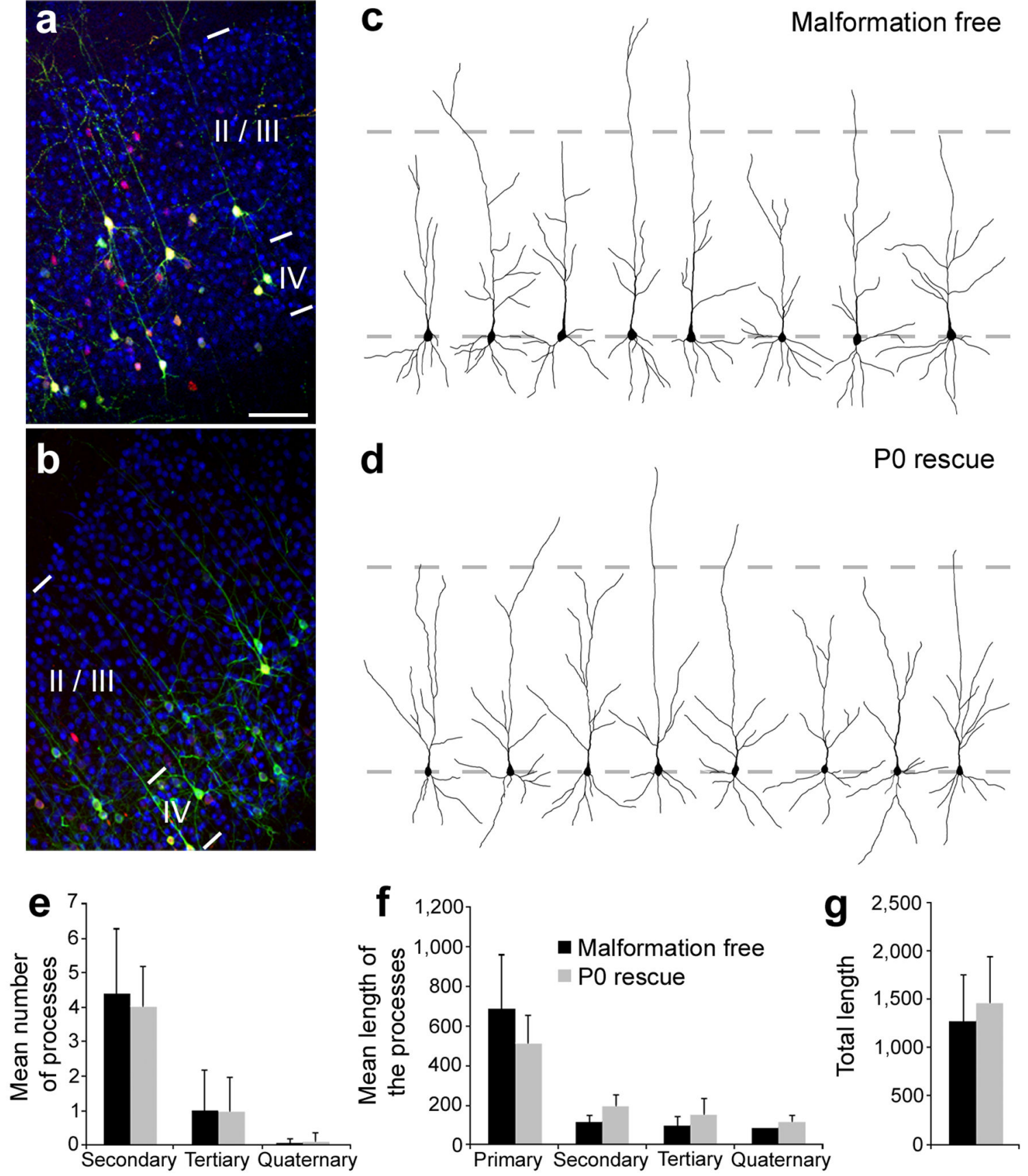

Figure 4. Morphology of rescued neurons

(a, b) Immunohistochemistry for the upper layers neurons marker CDP/Cux1 on P20 frontal neocortical sections. Animals were electroporated at E14 with either non-effective (3UTRm3hp) (a) or effective (3UTRhp) (b) Dcx-targeting shRNA vectors together with CAG-mRFP, CAG-ER ${ }^{\mathrm{T} 2} \mathrm{CreER}^{\mathrm{T} 2}$, and CALNL-eGFP (a) or CALNL-DCX-eGFP (b), and injected with 4-OHT at birth. Both initially correctly positioned transfected neurons (a) and initially mis-positioned transfected neurons induced to migrate to appropriated positions after Dcx re-expression (b) (both green and red) are located within the CDP/Cux 1+ band of 
upper layers neurons (in blue). (c, d) Reconstructed cortical neurons showing dendritic arborization in the same experimental conditions. (e-g) Quantifications of the dendritic arborization: mean number of apical processes per neurons (e), their mean length (f) and total length (g) (36-37 reconstructed neurons from 3-4 animals per condition). Scale bars, $200 \mu \mathrm{m}(\mathbf{a})$. 

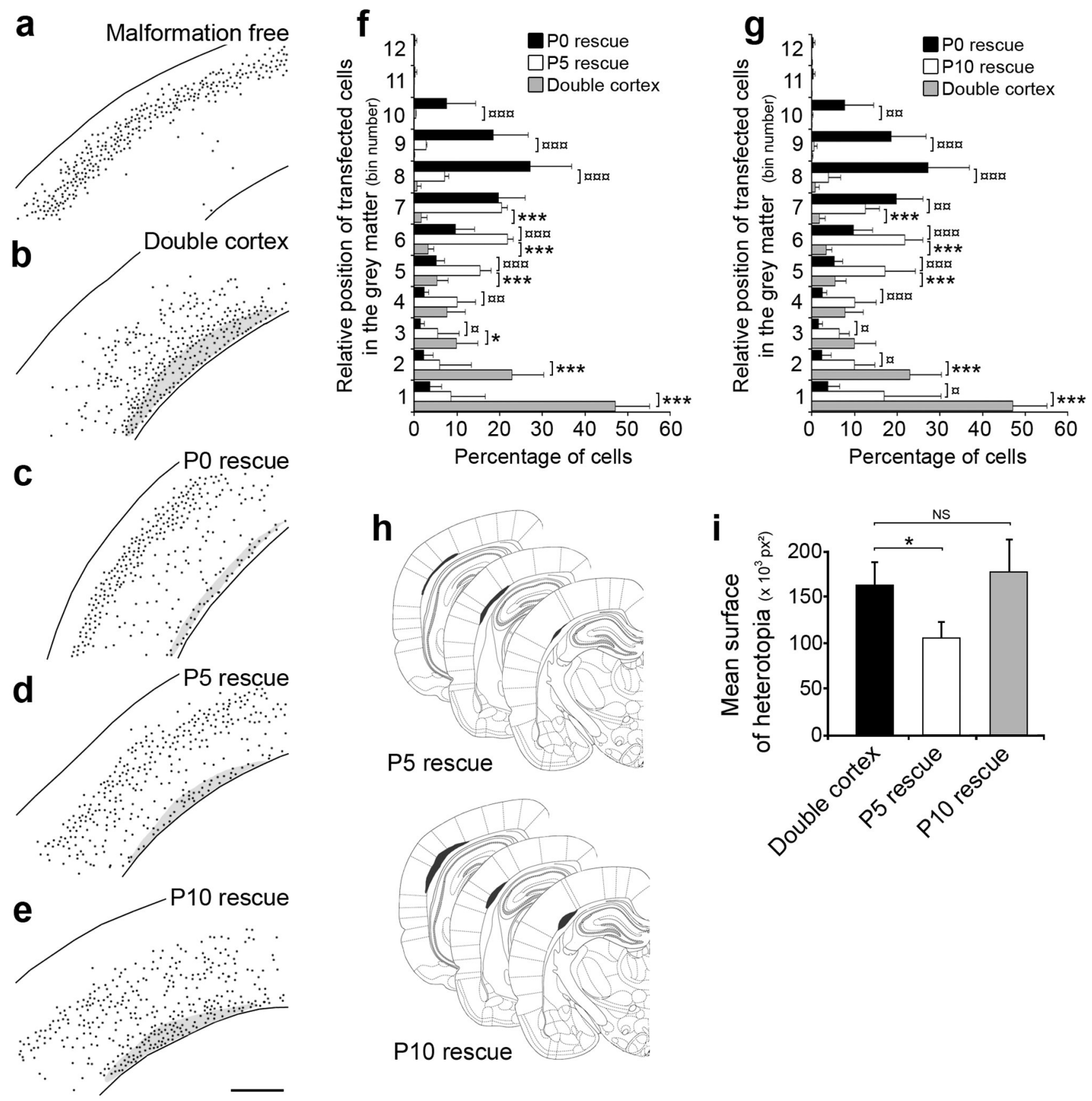

Figure 5. Critical developmental period for regressing malformation

(a-e) Representative reconstructed neocortical sections showing the laminar position of neurons at P20 after induction of eGFP $(\mathbf{a}, \mathbf{b})$ or DCX-eGFP expression at P0 (a-c), P5 (d) and P10 (e). Black dots represent positions of transfected cells. 4 plasmids were electroporated at E14: CAG-mRFP (a-e), CAG-ER ${ }^{\mathrm{T} 2} \mathrm{CreER}^{\mathrm{T} 2}(\mathbf{a}-\mathbf{e}), \mathrm{CALNL}-\mathrm{eGFP}(\mathbf{a}, \mathbf{b})$ or CALNL-DCX-eGFP (c-e), and 3UTRhp (c-e) or 3UTRm3hp (a), and 4-OHT was administered at P0 (a-c), P5 (d) and P10 (e). 
(f, g) Quantification of transfected cells distribution within the neocortical grey matter after induction of eGFP or DCX-eGFP expression at P5 (f) and P10 (g), compared to Dcx reexpression at P0 (8-12 sections from 2-3 animals per condition). (h) Size and position of SBH at 3 rostro-caudal levels after induction of Dcx expression at P5 (top) and P10 (bottom).

(i) Quantification of SBH surface after induction of Dcx expression at P5 and P10 (8 sections from 2-3 animals per condition).

*** $P<0.001, * * P<0.01, * P<0.05$. Scale bar, $500 \mu \mathrm{m}$. 
a

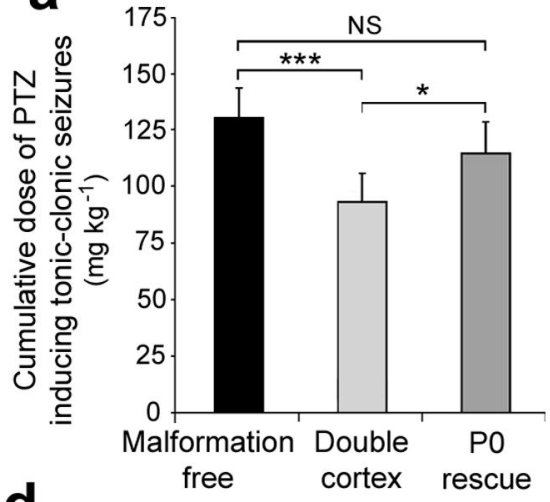

d
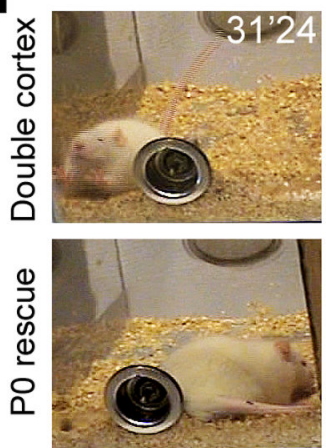

b
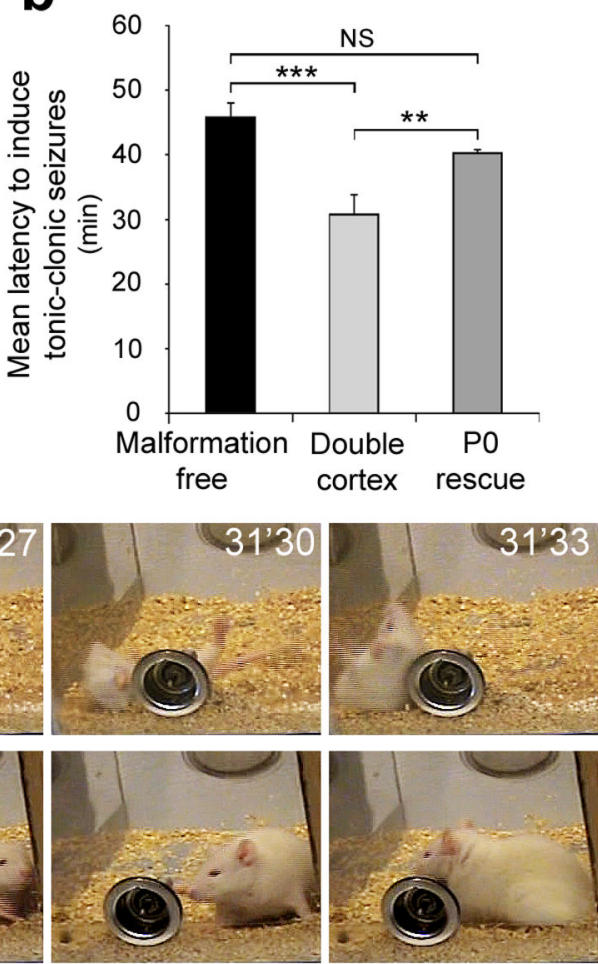

C
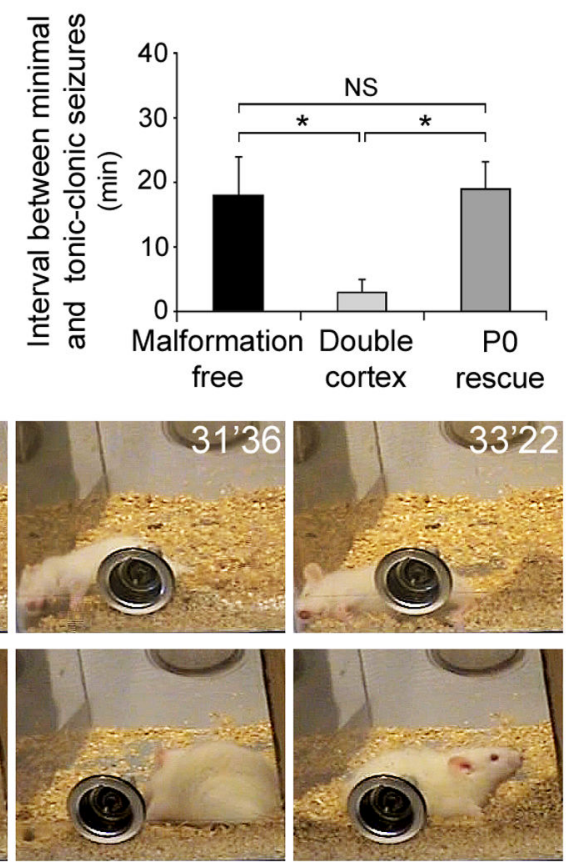

Figure 6. Decreased Seizure Susceptibility to PTZ-induced seizures

Quantification of doses of PTZ (a), latency to induce generalized tonic-clonic seizures (b) and interval between minimal and generalized seizures (c) in 3 groups of animals:

Malformation free animals (ineffective shRNAs and induced eGFP expression induced at P0), double cortex animals with SBH and lamination deficits (effective shRNAs and eGFP expression induced at $\mathrm{P} 0$ ) and $\mathrm{P} 0$ rescue animals with restored lamination and regressed SBH (effective shRNAs and DCX-eGFP expression induced at P0). (d) Example of a double cortex animal experiencing rearings, fallings and convulsions (top panels) and a P0 rescue animal at the same dose of PTZ and same time after injection (bottom panels). The P0 rescue animal experienced seizures 10 minutes later, after an additional injection of PTZ was given. $* * * P<0.001, * * P<0.01, * P<0.05$. 